ARTICLE

DOI: $10.1038 / s 41467-018-03251-7$

\title{
A quantum light-emitting diode for the standard telecom window around 1,550 nm
}

T. Müller ${ }^{1}$, J. Skiba-Szymanska ${ }^{1}$, A.B. Krysa $\mathbb{1}^{2}{ }^{2}$, J. Huwer ${ }^{1}$, M. Felle ${ }^{1,3}$, M. Anderson ${ }^{1,4}$, R.M. Stevenson ${ }^{1}$, J. Heffernan ${ }^{5}$, D.A. Ritchie (iD ${ }^{4} \&$ A.J. Shields ${ }^{1}$

Single photons and entangled photon pairs are a key resource of many quantum secure communication and quantum computation protocols, and non-Poissonian sources emitting in the low-loss wavelength region around 1,550 nm are essential for the development of fibrebased quantum network infrastructure. However, reaching this wavelength window has been challenging for semiconductor-based quantum light sources. Here we show that quantum dot devices based on indium phosphide are capable of electrically injected single photon emission in this wavelength region. Using the biexciton cascade mechanism, they also produce entangled photons with a fidelity of $87 \pm 4 \%$, sufficient for the application of one-way error correction protocols. The material system further allows for entangled photon generation up to an operating temperature of $93 \mathrm{~K}$. Our quantum photon source can be directly integrated with existing long distance quantum communication and cryptography systems, and provides a promising material platform for developing future quantum network hardware.

\footnotetext{
${ }^{1}$ Toshiba Research Europe Limited, 208 Science Park, Milton Road, Cambridge CB4 OGZ, UK. ${ }^{2}$ EPSRC National Epitaxy Facility, University of Sheffield, Sheffield S1 3JD, UK. ${ }^{3}$ Engineering Department Cambridge University, 9 J J Thomson Avenue, Cambridge CB3 OFA, UK. ${ }^{4}$ Cavendish Laboratory, University of Cambridge, J J Thomson Avenue, Cambridge CB3 OHE, UK. ${ }^{5}$ Department of Electronic and Electrical Engineering, University of Sheffield, Sheffield S1 3JD, UK. Correspondence and requests for materials should be addressed to T.Mül. (email: tina.muller@crl.toshiba.co.uk)
} 
Q uantum communication networks ${ }^{1}$ are expected to enable new applications such as cryptography secured by physical laws ${ }^{2}$, distributed quantum computing ${ }^{3}$ and networks of geographically remote clocks ${ }^{4}$. An essential building block for all these applications is a source of pure single photons and entangled pairs, compatible with the low-loss fibre telecom window around $1,550 \mathrm{~nm}$. Previous work based on gallium arsenide quantum dots ${ }^{5-7}$, colour centres in diamond ${ }^{8}$ or single atoms ${ }^{9}$ has been largely limited to wavelengths unsuitable for long distance fibre quantum network applications.

Quantum dots (QDs), semiconductor islands capable of confining charges in a discrete energy level structure, are particularly well-explored in the contexts of quantum communication and computing. Their implementation in InAs/GaAs, emitting around $900 \mathrm{~nm}$, has provided a rich physical system to demonstrate basic building blocks of a quantum network, such as individual entangled photon pairs from electrically driven devices ${ }^{5}$, photon sorters $^{6,7}$ and even entanglement between distant spins ${ }^{10,11}$. Further, in contrast to downconversion sources or strongly attenuated laser pulses, their photon emission follows sub-Poissonian statistics, a pre-requisite for the most efficient quantum cryptography protocols. However, although efforts have been made to extend their emission range to longer wavelengths ${ }^{12-15}$, electrically driven quantum light emission from QDs in the ideal telecommunication window around $1,550 \mathrm{~nm}$ has not been possible yet.

On the other hand, InP-based devices such as QD lasers readily reach the 1,550 $\mathrm{nm}$ telecom window. For quantum devices, the challenge is to enable optoelectronic access to individual dots that produce light with quantum signatures, i.e., single or entangled photons. In addition, although single photon emission has been demonstrated under optical excitation from InAs/InP dots grown by molecular beam epitaxy in the well-explored Stranski-Krastanow mode ${ }^{16-22}$, the resulting structures are often asymmetric dashes or horns, preventing access to the low intrinsic energy splitting of the photon polarisation states ${ }^{23}$ needed for entangled photon generation.

Recently, it has been shown that QD growth using metalorganic vapour phase epitaxy (MOVPE), which is the industry favoured growth method, can create droplet QDs with low fine structure splitting (FSS) ${ }^{24}$. Here we extend this growth scheme to produce the first optoelectronic devices for single and entangled photon emission in the $1,550 \mathrm{~nm}$ telecom window. Furthermore, we extend the working temperature up to $93 \mathrm{~K}$, allowing operation with liquid nitrogen or closed-cycle coolers.

\section{Results}

Device characterisation. The key features of our device are described in the cartoon in Fig. 1a, with a detailed fabrication description given in Methods. The structure is started with a 20repeats $\mathrm{n}$-doped distributed Bragg reflector (DBR) grown on the (001) surface, overgrown with a $3 / 4-\lambda$ intrinsic InP layer, upon which metallic In is deposited and droplets are formed on the surface (I.). The droplets are crystallised under $\mathrm{AsH}_{3}$ flow (II.) and overgrown with a $5 / 4-\lambda$ intrinsic InP region. The device is finished by a three-repeat p-doped Bragg reflector to complete an asymmetric $2-\lambda$ cavity for enhanced photon extraction (III.). For optoelectronic operation, mesas are wet-etched to the n-doped layer, and the $\mathrm{n}$ - and $\mathrm{p}$-doped layers are contacted using AuGeNi

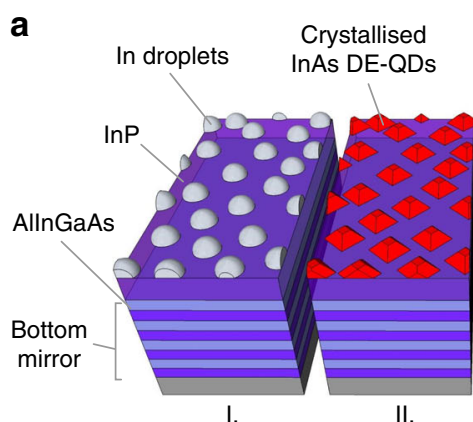

I.

C

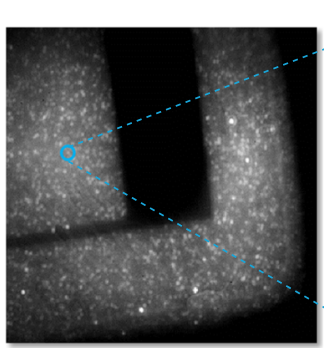

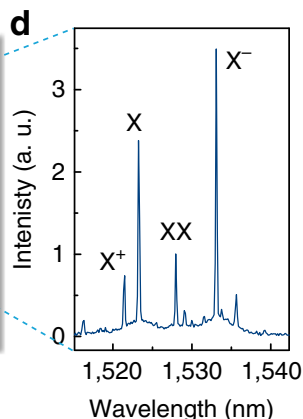

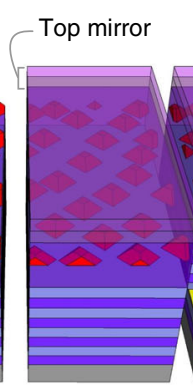

III. e

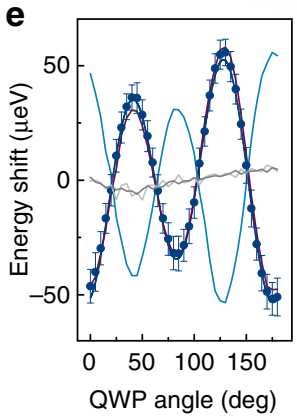

b top gate

$\mathrm{CrAu}$
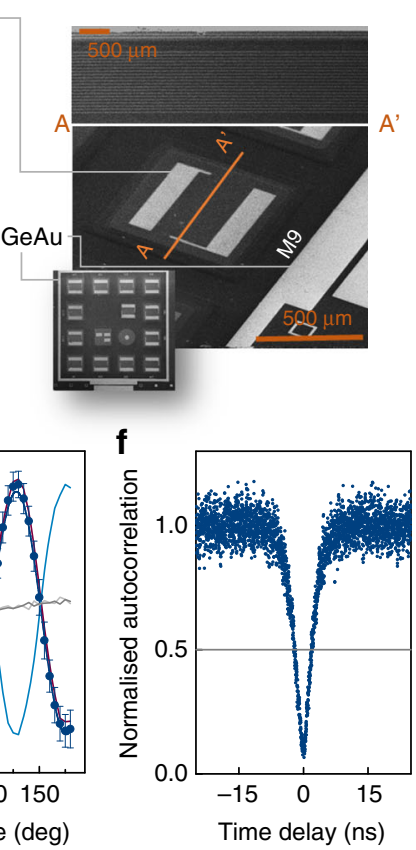

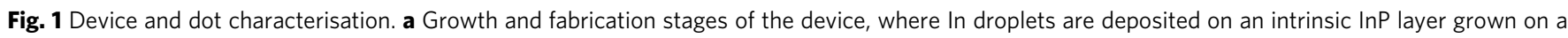
20-repeats doped Bragg mirror (I.), crystallised under As flux (II.) and overgrown by an intrinsic InP layer followed by a 3-repeats Bragg reflector (III.). Finally, mesas are wet-etched and the doped layers gated (IV.). b SEM image of a typical device mesa, with a cross-section through a mesa and a device

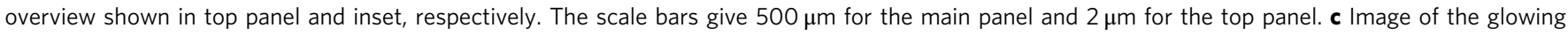
device taken with an InGaAs camera. The bright spots are individual QDs. d Spectrum of the QD circled in c with exciton ( $X$ ), biexciton (XX), positively charged exciton $\left(\mathrm{X}^{+}\right)$and negatively charged exciton $\left(\mathrm{X}^{-}\right)$labelled. The broad, low-intensity features show the influence of phonon interaction at $44 \mathrm{~K}^{35}$. $\mathbf{e}$ Energy shift of the $X$ (red curve), $X X$ (light blue curve), $X^{+}$(light grey curve) and $X^{-}$(grey curve) transitions as a function of quarter wave plate (QWP) angle (see main text). The transition energy at each QWP angle was determined as the centre energy of a Gaussian fit to the spectrum. The solid blue dots are the systematic shift-corrected X data points, with the error bars giving the SD derived from the covariance matrix of the Gaussian fit. The solid blue line gives the result of a fit to the QWP model described in Methods. $\mathbf{f}$ Second-order autocorrelation measurement performed on the X line in c 
a
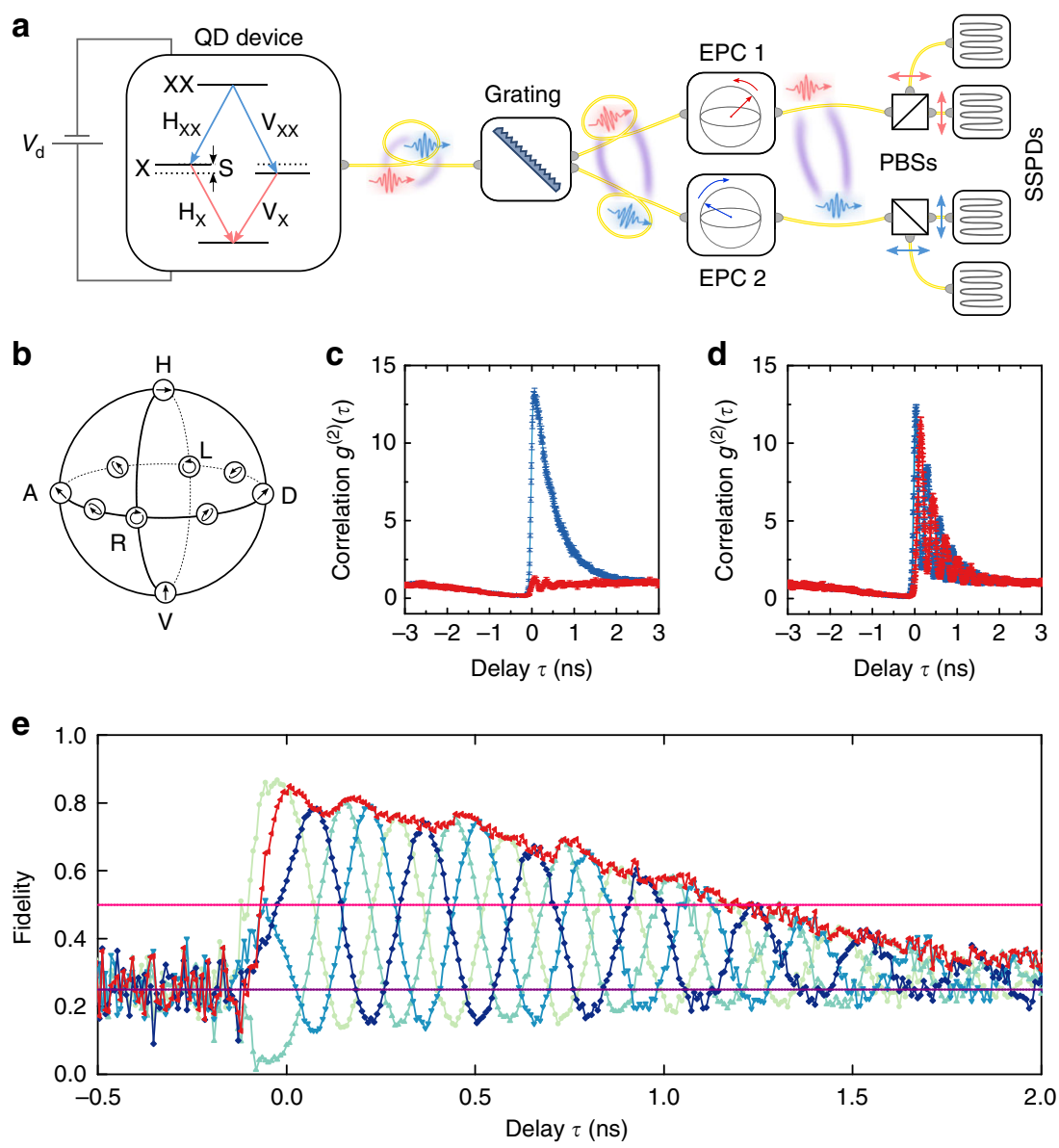

Fig. 2 Measurement of entanglement fidelity. a Schematic drawing of the entanglement setup. The entangled photons are generated by the biexciton cascade shown diagrammatically in the first panel. $X$ and XX photons are separated by a diffraction grating and sent to their respective polarisation sensitive detection units. These consist of electronic polarisation controllers (EPCs) and polarising beam splitters (PBSs) to prepare the detection system in one of five measurement bases. Polarisation selected photons are measured using superconducting single photon detectors (SSPDs). b Schematic of the Poincaré sphere, with measured bases indicated by cartoons of the photon polarisation. c, $\mathbf{d}$ Co-polarised (blue curves) and cross-polarised (red curves) biexciton-exciton photon coincidences measured in the HV and DA bases, respectively. e Entanglement fidelity to four maximally entangled states with phases $\chi=0, \pi / 2, \pi$ and $3 \pi / 2$ (light to dark blue curves), as well as to an evolving state (red curve). Pink and purple lines give the classical limit and uncorrelated values for coincidences, respectively

and $\mathrm{CrAu}$, respectively (IV.). A finished mesa is depicted in the scanning electron microscopic image in Fig. 1b, with a crosssection in the top panel clearly showing the layered mirrors afforded by the (001) growth surface, sandwiching the intrinsic region and QD layer. An outline of the entire device comprising 14 square mesas is shown in the inset. The resulting diode structure produces QD electroluminescence (EL) when driven in forward bias $>1.5 \mathrm{~V}$.

We can discern individual dots in our confocal microscope system as bright spots on a camera image, as shown in Fig. 1c. A typical EL spectrum of such a dot consists of bright, sharp, wellisolated lines, shown in Fig. 1d. The exciton (X) and biexciton (XX) transitions, upon which quantum entangled light emission is based, can be identified by monitoring their transition energy as a function of detected linear polarisation: the finite exciton FSS of $17.7 \pm 0.02 \mu \mathrm{eV}$ for this dot results in a variation in emission energy as shown in Fig. 1e, where the quarter-wave plate method described in Methods was used. In contrast, the energy of charged transitions is independent of detected polarisation. Both the $\mathrm{X}$ and XX transitions have linewidths limited by the spectrometer resolution $(89 \mu \mathrm{eV})$ and, after being filtered and guided to our superconducting single photon detection (SSPD) system, result in count rates up to $200 \mathrm{kcps}$. Further details on the specifics of our setup and detector efficiencies are given in Methods.
To determine the quantum nature of the observed transitions, we performed intensity autocorrelation $\left(g^{(2)}\right)$ measurements, as shown in Fig. 1f for the exciton transition of the dot in Fig. 1d. The dip in coincidences for zero delay is well below 0.5 , with the measured $g^{(2)}(0)=0.11 \pm 0.02$, which proves emission from a true single photon source. This value does not include corrections due to dark and background counts or detector jitter and therefore gives an upper bound to multiphoton emission from our device. Even without further optimisation, it is suppressed by almost a factor 10 compared with a Poissonian photon source.

Entangled photon generation. Next, we show that we can generate entangled photons with our telecom-wavelength diode. We use the biexciton cascade mechanism ${ }^{25}$, with the measurement configuration schematically shown in Fig. $2 \mathrm{a}$ and described further in Methods. Starting out with the QD in the biexciton state, radiative recombination of a first electron-hole pair leaves the dot occupied by a single exciton. Owing to conservation of angular momentum, the spins of the remaining charge carriers are entangled with the polarisation of the emitted biexciton photon. Recombination of the exciton therefore produces a photon in a polarisation state predefined by the path taken during the first recombination step, and leads to the emission of entangled 
photons in the maximally entangled state ${ }^{13}$

$$
\left|\psi_{\mathrm{B}}(\tau)\right\rangle=1 / \sqrt{2}\left(\left|\mathrm{H}_{\mathrm{XX}} \mathrm{H}_{\mathrm{X}}\right\rangle+\exp (i S \tau / \hbar)\left|\mathrm{V}_{\mathrm{XX}} \mathrm{V}_{\mathrm{X}}\right\rangle\right) .
$$

The phase factor here is acquired during the time $\tau$ spent in the exciton state: the fine structure splitting $S$ between the two spin states leads to a time-dependent oscillating correlation between the polarisation of the two photons of the form $\cos ^{2}(S \tau / 2 \hbar)^{13}$.

For the dot presented in Fig. 1, the XX-X polarisation correlations measured in the horizontal/vertical (HV) dot eigenbasis are shown in Fig. 2c. For negative time delays we find the expected dip in coincidences, corresponding to the unlikely arrival of an X photon before a XX photon. For positive time delays, co-polarised coincidences at their maximum are more than 10 times stronger than an uncorrelated source and decay for increasing time delays. This can be understood as follows: subsequently emitted XX and X photons are only correlated as long as they stem from the same excitation cycle. If the time delay between the two photons becomes comparable to the natural lifetime of the $X$, or the timescale of any other mechanism destroying the $\mathrm{X}$ population such as re-excitation, the likelihood of measuring uncorrelated photons increases, until, for long delays, only uncorrelated photons remain. In contrast to the copolarised case, cross-polarised coincidences never significantly exceed the Poissonian value. The effect of the finite $S$ in this dot is evident in the polarisation coincidences measured in a superposition basis, as shown in Fig. $2 \mathrm{~d}$ for the example of the diagonal/antidiagonal (DA) basis. For positive delays, the coincidences follow the oscillatory pattern outlined above. For the same reasons as the $\mathrm{HV}$ case, the envelope decays for increasing delays.

To prove that emission from our device is entangled and determine the fidelity to an evolving Bell state ${ }^{13,26}$, we performed the same coincidence measurements in three additional bases: the right- and left-hand circularly polarised and two elliptical bases $\left(\mathrm{E}_{\mathrm{LD}} \mathrm{E}_{\mathrm{RA}}\right.$ and $\left.\mathrm{E}_{\mathrm{LA}} \mathrm{E}_{\mathrm{RD}}\right)$ as marked on the Poincaré sphere in Fig. 2b. For unpolarised dot emission, the degree of correlation $C_{m n}$ between the polarisation basis states $m$ and $n$ is derived by dividing the difference between orthogonal coincidences by their sum, which gives the contrast between co- and cross-polarised coincidences. The fidelity to the expected ideal evolving state is then given by ${ }^{13}$

$$
\begin{aligned}
& F(t)=\frac{1}{4} \\
& {\left[1+C_{\mathrm{HV}}+\left(C_{\mathrm{DA}}-C_{\mathrm{LR}}\right) \cos \left(\frac{S \tau}{\hbar}\right)+\left(C_{\mathrm{E}_{\mathrm{LD}} \mathrm{E}_{\mathrm{RA}}}-C_{\mathrm{E}_{\mathrm{LA}} \mathrm{E}_{\mathrm{RD}}}\right) \sin \left(\frac{S \tau}{h}\right)\right],}
\end{aligned}
$$

which also expresses the fidelity to static Bell states for fixed values of $\chi=\frac{S \tau}{\hbar}$. The experimental results are shown in Fig. 2e for both the evolving and the static Bell states. The fidelities of our two photon emission to all states clearly violate the limit of 0.5 attainable by a classical photon source. The maximum fidelity of $0.87 \pm 0.04$, more than 9 SD above the classical limit, is obtained for $\chi=0$, corresponding to the symmetric Bell state. For the evolving choice of basis, it is straightforward to see that entanglement persists for over a nanosecond, eliminating the need for high-resolution temporal post selection. This even holds for static measurement bases, where the effect of the FSS can be effectively erased by employing appropriate measurement techniques ${ }^{27,28}$. We also evaluated the corresponding Bell's parameters $^{29}$, where the classical limit of 2 was violated by all states and a maximum of $2.35 \pm 0.27$ was reached. For long time delays, the fidelity drops to the uncorrelated value of 0.25 , once exciton emission from separate excitation cycles begins to dominate.

For quantum communication applications, error correction protocols can compensate for the effects of non-perfect entangled photon sources. For all bases, the fidelity reaches above the threshold of $70 \%$, corresponding to a quantum bit error rate of $20 \%$, required for two-way protocols ${ }^{30}$. The entangled light source is therefore suitable for quantum communication protocols enabling secure data networks protected by the laws of physics. In addition, for one-way error correction protocols such as CASCADE to apply, the quantum bit error rate has to be below $11 \%^{31}$, which corresponds to an entanglement fidelity of $83.5 \%$. This limit is surpassed by both the symmetric and the evolving bases.

Generating entangled photons at elevated temperatures. Finally, we show that droplet epitaxy QDs based on InAs/InP are able to produce entangled photons even at elevated temperatures. Figure $3 \mathrm{a}$ shows the measured fidelity to an evolving Bell state, while increasing the temperature from $44 \mathrm{~K}$ to $99 \mathrm{~K}$, for a constant driving voltage of $1.8 \mathrm{~V}$. The extracted maximum fidelities for each temperature are given in Fig. $3 \mathrm{~b}$ and they remain above the classical limit of 0.5 up to a temperature of $93.6 \mathrm{~K}$. This indicates that the spin relaxation quenching observed in GaAs $\mathrm{QDs}^{32}$ does not strongly affect InP based dots and, consequently, the operating temperature of our entangled photon diode exceeds those obtained for GaAs devices ${ }^{33}$.

Two independent effects are limiting the entanglement fidelity for elevated temperatures in our system. For temperatures below around $78 \mathrm{~K}$, phonon broadening ${ }^{22}$ causes photons from transitions other than $\mathrm{X}$ and $\mathrm{XX}$ to be emitted at these transition wavelengths, leading to uncorrelated detection events. As an example, the spectral broadening at $63 \mathrm{~K}$ is shown in the inset to Fig. 3b. Increasing the temperature also leads to faster decay timescales of entanglement fidelity, as seen in Fig. 3a, and quantified by the Gaussian HWHM plotted in Fig. 3c. This is due to the combined reduction of the timescales involved in the biexciton dynamics, which is shown for the example of the $\mathrm{X}$ decay time measured from unpolarised $\mathrm{XX}-\mathrm{X}$ cross-correlations and shown also in Fig. 3c. It is noteworthy that the entanglement fidelity persists for longer than the exciton lifetime for all temperatures below $78 \mathrm{~K}$. This is an indication that uncorrelated events start to dominate only at timescales longer than the $\mathrm{X}$ decay time, and that other processes disruptive of $\mathrm{X}$ population, such as re-excitation to higher levels, must be present. Around 78 $\mathrm{K}$, the fidelity decay time becomes comparable to the $34 \mathrm{ps}$ timing resolution of our detectors [blue solid line in Fig. 3c]. The convolution with the Gaussian detector response then leads to an effective averaging and decrease of the maximum fidelity beyond the phonon broadening effect. Higher resolution detector electronics and pulsed operation, together with a diode structure optimised for a reduction in $\mathrm{X}^{+}$intensity, would therefore increase the operation temperature of an entangled photon lightemitting diode even further.

\section{Discussion}

The InAs/InP droplet epitaxy QD devices we introduce here are expected to have significant impact in the development of quantum network technology. Unlike their InAs/GaAs predecessors, their emission wavelength near $1,550 \mathrm{~nm}$ will crucially enable them to interface with established quantum key distribution technology and extend the reach of quantum networks with quantum relays and repeaters. Superior performance is anticipated compared with light sources based on optical nonlinearities, by suppression of intrinsic errors due to sub-Poissonian character 

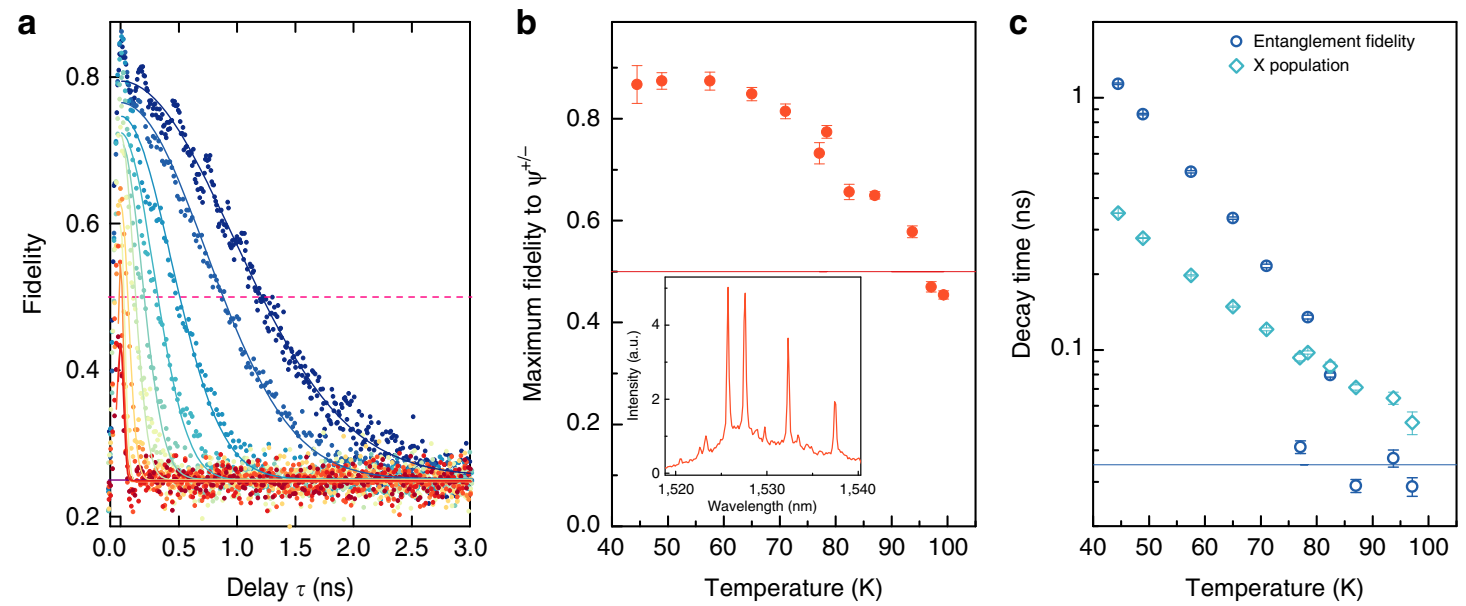

Fig. 3 Entanglement at elevated temperatures. a Fidelity to an evolving maximally entangled state when increasing the sample temperature from $44 \mathrm{~K}$ (dark blue) to $99 \mathrm{~K}$ (dark red) for a constant driving voltage of 1.8 V. Measured data (solid dots) are fitted with a Gaussian decay (solid curves) and the classical threshold of 0.5 is indicated by the dashed pink line. $\mathbf{b}$ Maximum fidelity to the $\psi^{+}$state as a function of temperature, where the error bars are the SD deduced from Poissonian statistics on the correlations. Again, the classical limit is indicated by the solid red line. Inset: dot spectrum at $63 \mathrm{~K}$. c Entanglement decay time constant extracted from the Gaussian fits in a (blue circles) and X decay time (turquoise diamonds), with the error bars giving the SD calculated from the fit covariance matrix. The half-width half-maximum (HWHM) of the detector timing resolution relevant for this measurement is around 30-34 ps, as shown by the solid blue line

of the emitted light. Pulsed operation of the devices is immediately feasible once integrated with suitable radio frequency electronics, as the physical mechanisms remain the same. Further, their compatibility with standard industry fabrication techniques, use of materials dominant in $1,550 \mathrm{~nm}$ photon detectors and amenability to miniaturisation and on-chip integration afforded by electrical operation makes them an attractive and practical building block for integrated quantum network transceivers, and engineering of the quantum internet.

\section{Methods}

Growth and fabrication of devices. On an InP substrate, we use MOVPE to grow 20 pairs of DBR, each pair consisting of $112 \mathrm{~nm}$ of $\left(\mathrm{Al}_{0.30} \mathrm{Ga}_{0.70}\right)_{0.48} \mathrm{In}_{0.52} \mathrm{As}$ and $123 \mathrm{~nm}$ of InP, both n-doped with Si (doping concentration $2 \times 10^{18} \mathrm{~cm}^{-3}$ ). After a $3 / 4-\lambda$ intrinsic InP layer, formation of In droplets was achieved by supply of In to the InP surface for $12 \mathrm{~s}$, via pyrolysis of trimethylindium at $400^{\circ} \mathrm{C}$, while withholding the supply of arsine to the growth chamber. The QD crystallisation process under arsine overpressure started at $400^{\circ} \mathrm{C}$ and carried on until the substrate reached $500^{\circ} \mathrm{C}$. Next, the dots were capped with $30 \mathrm{~nm}$ of InP followed by more InP at $640^{\circ} \mathrm{C}$. This resulted in a dot density $<10^{9} \mathrm{~cm}^{-2}$. A further $5 / 4-\lambda$ intrinsic InP was grown to cover the QDs before the cavity was finished off with three repeats of p-doped top DBR (doping concentration $2 \times 10^{18} \mathrm{~cm}^{-3}$ ). It is noteworthy that although the nominal dot composition is pure InAs, we can assume it is highly likely to be InAsP in reality, as the optical signal of a two-dimensional layer formed by As/P interchange during dot crystallisation is observed. To contact to the $\mathrm{n}$-doped layer, about $150 \mathrm{~nm}$ of AuGeNi slug was deposited and annealed at $375^{\circ} \mathrm{C}$. The p-doped layer was contacted by depositing around $20 \mathrm{~nm}$ of $\mathrm{Cr}$, followed by $100 \mathrm{~nm}$ of Au. A detailed characterisation of a similar dot growth protocol for undoped structures was reported earlier ${ }^{24}$.

Fibre-based confocal microscope. The device is placed in a temperature controlled environment in a He vapour cryostat and navigation to a desired dot is achieved through an imaging system using an InGaAs camera, which is highly sensitive in the near-infrared region and piezo-driven $x y z$ stages. Photons are collected using a confocal microscope with a high-numerical-aperture (NA) lens $(\mathrm{NA}=0.68)$ and coupled to a single-mode fibre that acts as a pinhole. The light is then guided either to a spectrometer or to a free-space grating setup to single out the biexction and exciton photons with a wavelength resolution $\sim 0.5 \mathrm{~nm}$. The light is then fibre-coupled again and sent to electrical polarisation controllers to prepare the light in one of the five basis states, before being polarisation filtered at a polarising beam splitter and sent to SSPDs with efficiencies ranging from $45 \%$ to $60 \%$ and timing jitters between $60-68$ ps as determined from the full-width at halfmaximum of a Gaussian fit. Photon arrival times are registered and compared using a time interval analyser (smallest time bin $32 \mathrm{ps}$ ).
QD transition identification. Neutral excitons and biexcitons are easily assigned a transition through the FSS measurement described in the main text. Positively and negatively charged excitons are identified by the behaviour at higher temperatures, where the positively charged exciton becomes more prominent as the limiting holes are supplied at greater abundance for elevated temperatures. Other less intense lines are due to further charged transitions as well as other dots nearby.

FSS measurement. Our method uses a quarter wave plate and subsequent polariser directly in front of the spectrometer, as opposed to the more commonly used half wave plate/polariser combination. It has the advantage that elliptical polarisations can be detected, which allows us to correct for birefringence in our setup optics. The measured energy deviation $\Delta E$ from the mean energy $\varepsilon$ of a state with polarisation $p$ (typically zero) as a function of the quarter wave plate angle $\chi$, with fitting parameters $\theta$ and $\phi$ describing the polarisation rotation and acquired phase shift caused by the setup optics, respectively, is given by ${ }^{24}$

$$
\begin{gathered}
\Delta E(\chi)=E(\chi)-\varepsilon=\frac{s}{2} \\
\left(\frac{2 p+\cos \theta(1+\cos 4 \chi)+\sin \theta \sin 4 \chi \cos \phi-2 \sin \theta \sin 2 \chi \sin \phi}{2+p \cos \theta(1+\cos 4 \chi)+p \sin \theta \sin 4 \chi \cos \phi-2 p \sin \theta \sin 2 \chi \sin \phi}\right) .
\end{gathered}
$$

Calibration of the polarisation states. For the calibration of the electronic polarisation controllers, a polarised light source was used to mimic QD emission. Its polarisation was aligned with the QD axes for the calibration of the HV basis and then set to the desired basis using a half wave plate followed by a quarter wave plate. The electronic polarisation controllers were independently varied in each basis to minimise transmission through the polarising beam splitters, effectively mapping the desired basis to the HV system. Co- and cross-polarised measurements were then taken using the transmission and reflection arms of the fibre based beam splitter. In practice, small deviations in the EPC alignment from the QD bases can occur, which are responsible for small admixtures of photons measured in bases other than the intended. This is the reason that remaining signatures of evolving states (oscillations) are observed in the HV correlations in Fig. 2c.

Data availability. The data that support the findings of this study are available on the Cambridge research repository Apollo with the identifier DOI 10.17863/ CAM. $17330^{34}$.

Received: 26 September 2017 Accepted: 29 January 2018 Published online: 28 February 2018

\section{References}

1. Kimble, H. J. The quantum internet. Nature 453, 1023-1030 (2008).

2. Scarani, V. et al. The security of practical quantum key distribution. Rev. Mod. Phys. 81, 1301-1350 (2009).

3. Cirac, J. I., Ekert, A. K., Huelga, S. F. \& Macchiavello, C. Distributed quantum computation over noisy channels. Phys. Rev. A 59, 4249-4254 (1999). 
4. Kómár, P. et al. A quantum network of clocks. Nat. Phys. 10, 582-587 (2014).

5. Salter, C. L. et al. An entangled-light-emitting diode. Nature 465, 594-597 (2010).

6. Bennett, A. J. et al. A semiconductor photon-sorter. Nat. Nanotechnol. 11, 857-860 (2016).

7. De Santis, L. et al. A solid-state single-photon filter. Nat. Nanotechnol. 12, 663-667 (2017).

8. Kalb, N. et al. Entanglement distillation between solid-state quantum network nodes. Science 356, 928-932 (2017).

9. Hacker, B., Welte, S., Rempe, G. \& Ritter, S. A photon-photon quantum gate based on a single atom in an optical resonator. Nature 536, 193-196 (2016).

10. Delteil, A. et al. Generation of heralded entanglement between distant hole spins. Nat. Phys. 12, 218-223 (2016).

11. Stockill, R. et al. Phase-tuned entangled state generation between distant spin qubits. Phys. Rev. Lett. 119, 010503 (2017).

12. Zinoni, C. et al. Time-resolved and antibunching experiments on single quantum dots at $1300 \mathrm{~nm}$. Appl. Phys. Lett. 88, 131102 (2006).

13. Ward, M. B. et al. Coherent dynamics of a telecom-wavelength entangled photon source. Nat. Commun. 5, 3316 (2014).

14. Kettler, J. et al. Neutral and charged biexciton-exciton cascade in neartelecom-wavelength quantum dots. Phys. Rev. B 94, 045303 (2016).

15. Paul, M. et al. Single-photon emission at $1.55 \mu \mathrm{m}$ from MOVPE-grown InAs quantum dots on InGaAs/GaAs metamorphic buffers. Appl. Phys. Lett. 111, 033102 (2017)

16. Kim, D. et al. Photoluminescence of single, site-selected, InAsInP quantum dots in high magnetic fields. Appl. Phys. Lett. 87, 212105 (2005).

17. Cade, N. I., Gotoh, H., Kamada, H. \& Nakano, H. Optical characteristics of single InAs/nGaAsP/nP(100) quantum dots emitting at $1.55 \mu \mathrm{m}$. Appl. Phys. Lett. 89, 181113 (2006)

18. Takemoto, K., Takatsu, M., Hirose, S. \& Yokoyama, N. An optical horn structure for single-photon source using quantum dots at telecommunication wavelength. J. Appl. Phys. 101, 081720 (2007).

19. Dalacu, D. et al. Deterministic emitter-cavity coupling using a single-site controlled quantum dot. Phys. Rev. B 82, 033301 (2010).

20. Benyoucef, M., Yacob, M., Reithmaier, J. P., Kettler, J. \& Michler, P. Telecomwavelength $(1.5 \mu \mathrm{m})$ single-photon emission from InP-based quantum dots. Appl. Phys. Lett. 103, 162101 (2013).

21. Dusanowski, Ł. et al. Single photon emission at $1.55 \mu \mathrm{m}$ from charged and neutral exciton confined in a single quantum dash. Appl. Phys. Lett. 105, 021909 (2014)

22. Takemoto, K. et al. Quantum key distribution over $120 \mathrm{~km}$ using ultrahigh purity single-photon source and superconducting single-photon detectors. Sci. Rep. 5, 14383 (2015).

23. He, L., Gong, M., Li, C.-F., Guo, G.-C. \& Zunger, A. Highly reduced finestructure splitting in InAs/InP quantum dots offering an efficient on-demand entangled 1.55- $\mu \mathrm{m}$ photon emitter. Phys. Rev. Lett. 101, 157405 (2008).

24. Skiba-Szymanska, J. et al. Universal growth scheme for quantum dots with low fine-structure splitting at various emission wavelengths. Phys. Rev. Appl. 8, 014013 (2017).

25. Benson, O., Santori, C., Pelton, M. \& Yamamoto, Y. Regulated and entangled photons from a single quantum dot. Phys. Rev. Lett. 84, 2513-2516 (2000).

26. Stevenson, R. M. et al. Evolution of entanglement between distinguishable light states. Phys. Rev. Lett. 101, 170501 (2008).

27. Wang, X.-B., Yang, C.-X. \& Liu, Y.-B. On-demand entanglement source with polarization-dependent frequency shift. Appl. Phys. Lett. 96, 201103 (2010).

28. Fognini, A., Ahmadi, A., Daley, S. J., Reimer, M. E. \& Zwiller, V. Universal finestructure eraser for quantum dots. Preprint at http://arxiv.org/abs/ 1707.04104 (2017).
29. Clauser, J. F., Horne, M. A., Shimony, A. \& Holt, R. A. Proposed experiment to test local hidden-variable theories. Phys. Rev. Lett. 23, 880-884 (1969).

30. Chau, H. F. Practical scheme to share a secret key through a quantum channel with a $27.6 \%$ bit error rate. Phys. Rev. A. 66, 060302 (2002).

31. Brassard, G. \& Savail, L. Advances in Cryptology - EUROCRYPT '93. in Advances in Cryptology-EUROCRYPT '93: Lecture Notes in Computer Science (ed. Helleseth, T.) 765, 410-423 (Springer, 1994).

32. Paillard, M. et al. Spin relaxation quenching in sSemiconductor quantum dots. Phys. Rev. Lett. 86, 1634-1637 (2001).

33. Dousse, A. et al. A quantum dot based bright source of entangled photon pairs operating at $53 \mathrm{~K}$. Appl. Phys. Lett. 97, 081104 (2010).

34. Müller, T. et al. Research data supporting a quantum light emitting diode for the standard telecom window around 1550nm. Cambridge Research Repository Apollo. https://doi.org/10.17863/CAM.17330 (2018).

35. Besombes, L., Kheng, K., Marsal, L. \& Mariette, H. Acoustic phonon broadening mechanism in single quantum dot emission. Phys. Rev. B 63, 155307 (2001).

\section{Acknowledgements}

We thank Marco Lucamarini, Anthony Bennett and Peter Spencer for discussions. This work has been co-funded by the UK's innovation agency, Innovate UK and the EPSRC.

\section{Author contributions}

A.J.S., R.M.S., D.A.R. and J. Heffernan conceived the experiment and supervised the project. J.S.-S., T.M., J. Huwer, A.B.K. and R.M.S. designed the QD structures. A.B.K. engineered the epitaxial process, grew the diode wafers and performed basic character isation of the epitaxial material. J.S.-S. coordinated growth and manufactured the device T.M., J. Huwer, M.F. and M.A. took the measurements and analysed the data. T.M., R.M.S. and A.J.S. wrote the manuscript. All authors discussed the results and commented on the manuscript.

\section{Additional information}

Competing interests: The authors declare no competing interests.

Reprints and permission information is available online at http://npg.nature.com/ reprintsandpermissions/

Publisher's note: Springer Nature remains neutral with regard to jurisdictional claims in published maps and institutional affiliations.

(i) Open Access This article is licensed under a Creative Common Attribution 4.0 International License, which permits use, sharing, adaptation, distribution and reproduction in any medium or format, as long as you give appropriate credit to the original author(s) and the source, provide a link to the Creative Commons license, and indicate if changes were made. The images or other third party material in this article are included in the article's Creative Commons license, unless indicated otherwise in a credit line to the material. If material is not included in the article's Creative Commons license and your intended use is not permitted by statutory regulation or exceeds the permitted use, you will need to obtain permission directly from the copyright holder. To view a copy of this license, visit http://creativecommons.org/ licenses/by/4.0/.

(c) The Author(s) 2018 